\title{
Compound Difference Anti-Synchronization between Hyper-Chaotic Systems of Fractional Order
}

\author{
A. Khan, L. S. Jahanzaib, Nasreen, P. Trikha*, T. Khan \\ Department of Mathematics, Jamia Millia Islamia, New Delhi, India
}

Received 20 October 2019, accepted in final revised form 6 January 2020

\begin{abstract}
In this article, the compound difference anti-synchronization between fractional order hyper-chaotic systems have been studied. Numerical simulations have been performed using MATLAB to verify the theoretical results on fractional order Xling, Vanderpol, Rikitake and Rabinovich hyper-chaotic systems.

Keywords: Compound difference anti-synchronization; Xling system; Vanderpol system; Rikitake system; Rabinovich system.

(C) 2020 JSR Publications. ISSN: 2070-0237 (Print); 2070-0245 (Online). All rights reserved. doi: http://dx.doi.org/10.3329/jsr.v12i2.43764 J. Sci. Res. 12 (2), 175-181 (2020)
\end{abstract}

\section{Introduction}

CHAOS theory has been gaining popularity ever since the well-known Lorenz system was discovered. From then on, there has been no looking back in the growth and development of chaos theory. Chaos synchronization [1] was introduced by Pecora and Carroll in 1990.In synchronization two chaotic systems arising from different initial conditions is made stable by designing controllers. Where synchronizing [2-5] two chaotic systems is considered difficult, synchronizing more than two hyper-chaotic systems is in itself a big challenge.

Though fractional calculus is not new to mathematics, it has recently emerged most useful in modelling of processes and systems where integer order could not serve purpose. Motivated by the above discussions hyper-chaotic systems have been synchronized here. Numerical Simulations have been performed using MATLAB which verify the theoretical results.

\section{Problem Formulation}

Consider three hyper-chaotic fractional order master systems and one chaotic fractional order slave system we formulate the compound difference anti-synchronization scheme. Let the scaling master system be:

${ }^{*}$ Corresponding author: pushali.t@gmail.com 


$$
D^{q} x=f_{1}(x)
$$

Let the base master systems be:

$$
\begin{aligned}
& D^{q} y=f_{2}(y) \\
& D^{q} z=f_{3}(z)
\end{aligned}
$$

Let the slave system be:

$$
D^{q} w=f_{4}(w)+v
$$

Where $\mathrm{x}=\left(x_{1}, x_{2}, \ldots, x_{n}\right), \mathrm{y}=\left(y_{1}, y_{2}, \ldots, y_{n}\right), \mathrm{z}=\left(z_{1}, z_{2}, \ldots, z_{n}\right)$ and $\mathrm{w}=\left(w_{1}, w_{2}, \ldots, w_{n}\right)$ are state vectors of the respective systems, $f_{i}, \mathrm{i}=1,2,3,4$ are continuous functions, $0<q<1$ and $v=\left(v_{1}, v_{2}, \ldots, v_{n}\right)$, is controller to be designed.

Defining the error as:

$$
\mathrm{e}=\mathrm{Aw}+\mathrm{Bx}(\mathrm{Cz}-\mathrm{Dy})
$$

where $A=\operatorname{diag}\left(\alpha_{1}, \alpha_{2}, \ldots, \alpha_{n}\right), \quad B=\operatorname{diag}\left(\beta_{1}, \beta_{2}, \ldots, \beta_{n}\right), \quad C=\operatorname{diag}\left(\gamma_{1}, \gamma_{2}, \ldots, \gamma_{n}\right)$, $D=\operatorname{diag}\left(\delta_{1}, \delta_{2}, \ldots, \delta_{n}\right)$ are suitably chosen diagonal matrices with $A \neq 0$.

To achieve the desired anti-synchronization we must have error tending to zero, i.e.

$$
\begin{gathered}
\operatorname{Lim}\|\mathrm{e}\|=0 \text { as } \mathrm{t} \rightarrow \infty \\
\text { i.e., } \operatorname{Lim}\|\mathrm{Aw}+\mathrm{Bx}(\mathrm{Cz}-\mathrm{Dy})\|=0 \text { as } \mathrm{t} \rightarrow \infty
\end{gathered}
$$

Where $\|$.$\| represents the Euclidean norm.$

We here define the controllers as:

$$
v_{i}=\frac{\Theta_{i}}{\alpha_{i}}-f_{4 i}-\frac{\mathrm{K}_{i} \mathrm{e}_{i}}{\alpha_{i}}
$$

Where $\Theta_{i}=\beta_{i} \mathrm{f}_{1 i}\left(\gamma_{i} \mathrm{z}_{i}-\delta_{i} \mathrm{y}_{i}\right)+\beta_{i} \mathrm{x}_{i}\left(\gamma_{i} \mathrm{f}_{3 i}-\delta_{i} \mathrm{f}_{4 i}\right)$ for $i=1,2, \ldots, n$

Theorem: Systems (1)-(3) will be in compound difference anti-synchronization with (4) if the controllers are designed as in (5).

Proof: We define the compound difference anti-synchronization error as:

$$
e_{i}=\alpha_{i} w_{i}+\beta_{i} x_{i}\left(\gamma_{i} z_{i}-\delta_{i} y_{i}\right)
$$

Differentiating (6) we get the error dynamical system as:

$$
D^{q} e_{i}=\alpha_{i} D^{q} w_{i}+\beta_{i} D^{q} x_{i}\left(\gamma_{i} z_{i}-\delta_{i} y_{i}\right)+\beta_{i} x_{i}\left(\gamma_{i} D^{q} z_{i}-\delta_{i} D^{q} y_{i}\right)
$$

Substituting the values of the derivatives and applying the designed controller, the error dynamical system simplifies to:

$$
D^{q} e_{i}=-K_{i} e_{i}
$$

Next, we consider the Lyapunov function as:

$$
\mathrm{V}(\mathrm{e}(\mathrm{t}))=\frac{1}{2}\left(e_{1}{ }^{2}+e_{2}{ }^{2}+e_{3}{ }^{2}+{e_{4}}^{2}\right)
$$

Differentiating we get

$$
D^{q}(V(e(t))) \leq\left(e_{1} D^{q} e_{1}+e_{2} D^{q} e_{2}+e_{3} D^{q} e_{3}+e_{4} D^{q} e_{4}\right)
$$

Substituting (8) into (10), we get

$$
\begin{aligned}
& D^{q}(\mathrm{~V}(\mathrm{e}(\mathrm{t}))) \leq\left(e_{1}\left(-K_{1} e_{1}\right)+e_{2}\left(-K_{2} e_{2}\right)+e_{3}\left(-K_{3} e_{3}\right)+e_{4}\left(-K_{4} e_{4}\right)\right) \\
& =-K_{1} e_{1}{ }^{2}-K_{2} e_{2}{ }^{2}-K_{3} e_{3}{ }^{2}-K_{4} e_{4}{ }^{2}
\end{aligned}
$$


i.e. $\mathrm{V}(\mathrm{e}(\mathrm{t}))$ is positive definite function with a negative definite derivative. Hence, by Lyapunov Stability Theory we have that error tends to zero, implying desired antisynchronization has been achieved.

Note: We have taken Caputo's version of fractional derivative in our paper.

\section{System Description}

\subsection{Scaling master system}

We consider the fractional order hyper-chaotic Xling system as the scaling master system given by:

Fractional Order Hyper-Chaotic Xling System

$$
\begin{aligned}
\frac{d^{q} x_{1}}{d t^{q}} & =a_{1}\left(x_{2}-x_{1}\right)+x_{4} \\
\frac{d^{q} x_{2}}{d t^{q}} & =a_{2} x_{1}+x_{1} x_{3}-x_{4} \\
\frac{d^{q} x_{3}}{d t^{q}} & =-a_{3} x_{3}-a_{4} x_{1}{ }^{2} \\
\frac{d^{q} x_{4}}{d t^{q}} & =a_{3} x_{1}
\end{aligned}
$$

Here $x=\left(x_{1}, x_{2}, x_{3}, x_{4}\right) \in \mathbb{R}^{4}$ are state variables and $a_{1}, a_{2}, a_{3}, a_{4} \in \mathbb{R}$ are parameters.

For parameter values $a_{1}=10, a_{2}=40, a_{3}=2.5, a_{4}=4$ and initial conditions of state variables as $(1,2,3,4)$ the system shows chaotic behavior as displayed in Fig. 1 (a).

\subsection{Base master systems}

Next we consider the hyperchaotic fractional order Rabinovich and Rikitake chaotic systems.

Fractional Order Hyper-Chaotic Rabinovich System

$$
\begin{aligned}
& \frac{d^{q} y_{1}}{d t^{q}}=-c_{1} y_{1}+c_{2} y_{2}+y_{2} y_{3} \\
& \frac{d^{q} y_{2}}{d t^{q}}=c_{2} y_{1}-y_{2}+y_{4}-y_{1} y_{3} \\
& \frac{d^{q} y_{3}}{d t^{q}}=-y_{3}+y_{1} y_{2} \\
& \frac{d^{q} y_{4}}{d t^{q}}=-c_{3} y_{2}
\end{aligned}
$$

Here $y=\left(y_{1}, y_{2}, y_{3}, y_{4}\right) \in \mathbb{R}^{4}$ are state variables and $c_{1}, c_{2}, c_{3} \in \mathbb{R}$ are parameters.

For parameter values $c_{1}=34, c_{2}=6.75, c_{3}=2$ and initial conditions of state variables as $(5.5,-1.25,8.4,2.75)$ the system shows chaotic behavior as displayed in Fig. 1 (b).

Fractional Order Hyper-Chaotic Rikitake System

$$
\begin{aligned}
\frac{d^{q} z_{1}}{d t^{q}} & =-z_{1}+z_{2} z_{3}-d_{1} z_{4} \\
\frac{d^{q} z_{2}}{d t^{q}} & =-z_{2}+z_{1}\left(z_{3}-d_{2}\right)-d_{1} z_{4} \\
\frac{d^{q} z_{3}}{d t^{q}} & =d_{2}-z_{1} z_{2} \\
\frac{d^{q} z_{4}}{d t^{q}} & =d_{3} z_{2}
\end{aligned}
$$


Here $\mathrm{z}=\left(z_{1}, z_{2}, z_{3}, z_{4}\right) \in \mathbb{R}^{4}$ are state variables and $d_{1}, d_{2}, d_{3} \in \mathbb{R}$ are parameters.

For parameter values $d_{1}=1.7, d_{2}=1, d_{3}=0.7$ and initial conditions of state variables as $(3.5,1.7,-4.5,2.8)$ the system shows chaotic behavior as displayed in Fig. 1 (c).

\subsection{Slave system}

We consider the slave system as the hyperchaotic fractional order Vanderpol system.

Fractional Order Hyper-Chaotic Vanderpol System

$$
\begin{aligned}
\frac{d^{q} w_{1}}{d t^{q}} & =w_{2} \\
\frac{d^{q} w_{2}}{d t^{q}} & =-\left(b_{1}+b_{2} w_{3}\right) w_{1}-\left(b_{1}+b_{2} w_{3}\right) w_{1}^{3}-b_{3} w_{2}+b_{4} w_{3} \\
\frac{d^{q} w_{3}}{d t^{q}} & =w_{4} \\
\frac{d^{q} w_{4}}{d t^{q}} & =-w_{3}+b_{6} w_{1}+b_{5} w_{4}\left(1-w_{3}^{2}\right)
\end{aligned}
$$

Here $w=\left(w_{1}, w_{2}, w_{3}, w_{4}\right) \in \mathbb{R}^{4}$ are state variables and $b_{1}, b_{2}, b_{3}, b_{4}, b_{5}, b_{6} \in \mathbb{R}$ are parameters.

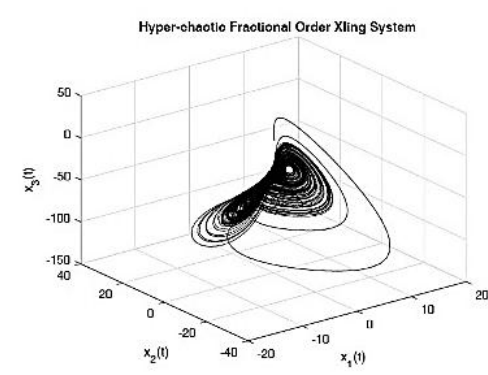

(a)

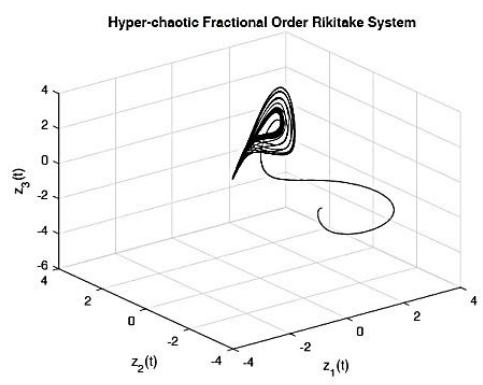

(c)

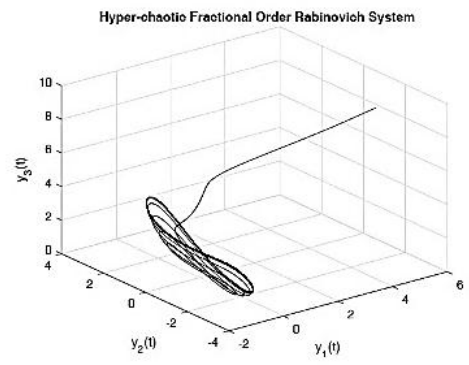

(b)

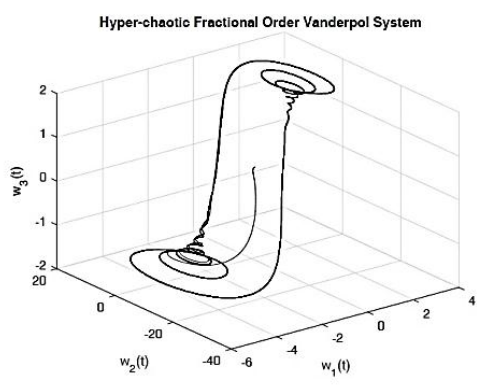

(d)

Fig. 1. Phase portraits of the scaling master system, base master system I, base master system -II, slave system in (a) $x_{1}-x_{2}-x_{3}$ space, (b) $y_{1}-y_{2}-y_{3}$ space, (c) $z_{1}-z_{2}-z_{3}$ space and (d) $w_{1}-w_{2}-w_{3}$ space 
For parameter values $b_{1}=10, b_{2}=3, b_{3}=0.4, b_{4}=70, b_{5}=5, b_{6}=0.1$ and initial conditions of state variables as $(0.1,-0.5,0.1,-0.5)$ the system shows chaotic behavior as displayed in Fig. 1 (d).

\section{Compound Difference Anti-Synchronization between Hyperchaoticsystems of Fractional Orders}

\subsection{Numerical simulations and discussions}

Corresponding to master system (1)-(3) and slave system (4), the slave system with control functions is given as:

$$
\begin{aligned}
& \frac{d^{q} w_{1}}{d t^{q}}=w_{2}+v_{1} \\
& \frac{d^{q} w_{2}}{d t^{q}}=-\left(b_{1}+b_{2} w_{3}\right) w_{1}-\left(b_{1}+b_{2} w_{3}\right) w_{1}^{3}-b_{3} w_{2}+b_{4} w_{3}+v_{2} \\
& \frac{d^{q} w_{3}}{d t^{q}}=w_{4}+v_{3} \\
& \frac{d^{q} w_{4}}{d t^{q}}=-w_{3}+b_{6} w_{1}+b_{5} w_{4}\left(1-w_{3}^{2}\right)+v_{4}
\end{aligned}
$$

We define the error given by (6) as:

$$
\begin{aligned}
& e_{1}=\alpha_{1} w_{1}+\beta_{1} x_{1}\left(\gamma_{1} z_{1}-\delta_{1} y_{1}\right) \\
& e_{2}=\alpha_{2} w_{2}+\beta_{2} x_{2}\left(\gamma_{2} z_{2}-\delta_{2} y_{2}\right) \\
& e_{3}=\alpha_{3} w_{3}+\beta_{3} x_{3}\left(\gamma_{3} z_{3}-\delta_{3} y_{3}\right) \\
& e_{4}=\alpha_{4} w_{4}+\beta_{4} x_{4}\left(\gamma_{4} z_{4}-\delta_{4} y_{4}\right)
\end{aligned}
$$



(a)

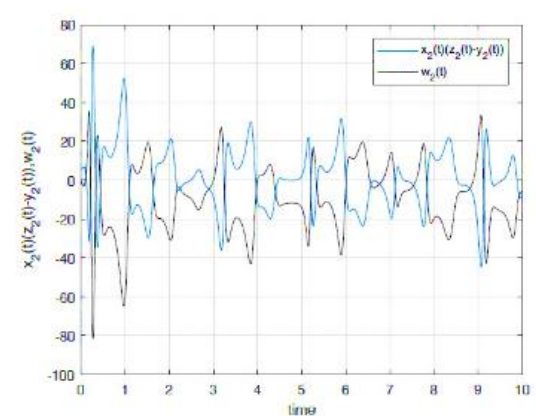

(b) 


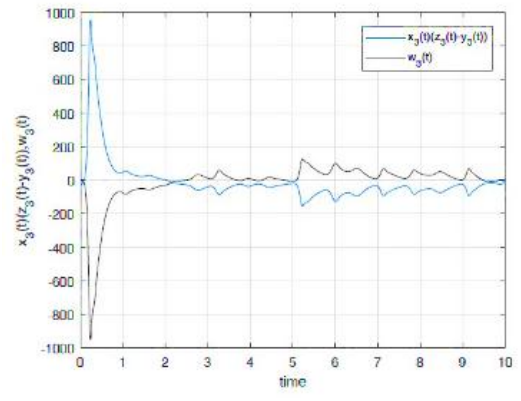

(c)

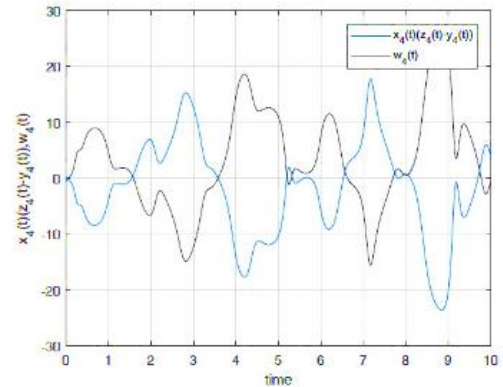

(d)

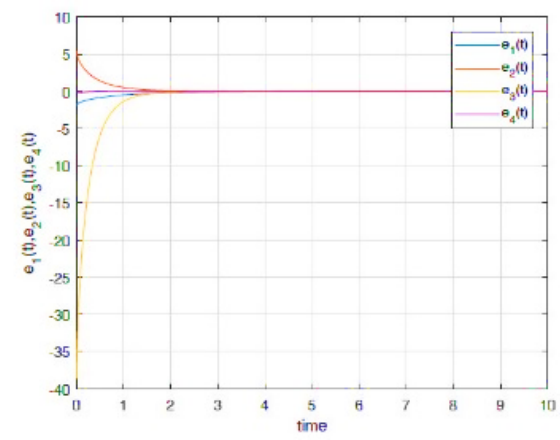

(e)

Fig. 2. Anti-synchronized trajectories of compound of master systems with slave system and error plot.

The error dynamical system is given by:

$$
\begin{gathered}
\frac{d^{q} w_{1}}{d t^{q}}=\alpha_{1} \frac{d^{q} w_{1}}{d t^{q}}+\beta_{1} \frac{d^{q} x_{1}}{d t^{q}}\left(\gamma_{1} z_{1}-\delta_{1} y_{1}\right)+\beta_{1} x_{1}\left(\gamma_{1} \frac{d^{q} z_{1}}{d t^{q}}-\delta_{1} \frac{d^{q} y_{1}}{d t^{q}}\right) \\
\frac{d^{q} w_{2}}{d t^{q}}=\alpha_{2} \frac{d^{q} w_{2}}{d t^{q}}+\beta_{2} \frac{d^{q} x_{2}}{d t^{q}}\left(\gamma_{2} z_{2}-\delta_{2} y_{2}\right)+\beta_{2} x_{2}\left(\gamma_{2} \frac{d^{q} z_{2}}{d t^{q}}-\delta_{2} \frac{d^{q} y_{2}}{d t^{q}}\right) \\
\frac{d^{q} w_{3}}{d t^{q}}=\alpha_{3} \frac{d^{q} w_{3}}{d t^{q}}+\beta_{3} \frac{d^{q} x_{3}}{d t^{q}}\left(\gamma_{3} z_{3}-\delta_{3} y_{3}\right)+\beta_{3} x_{3}\left(\gamma_{3} \frac{d^{q} z_{3}}{d t^{q}}-\delta_{3} \frac{d^{q} y_{3}}{d t^{q}}\right) \\
\frac{d^{q} w_{1}}{d t^{q}}=\alpha_{4} \frac{d^{q} w_{1}}{d t^{q}}+\beta_{4} \frac{d^{q} x_{4}}{d t^{q}}\left(\gamma_{4} z_{4}-\delta_{4} y_{4}\right)+\beta_{4} x_{4}\left(\gamma_{4} \frac{d^{q} z_{4}}{d t^{q}}-\delta_{4} \frac{d^{q} y_{4}}{d t^{q}}\right)
\end{gathered}
$$

Substituting values of the derivatives from (12)-(15) and designing controllers as:

$$
v_{1}=\frac{\Theta_{1}}{\alpha_{1}}-f_{41}-\frac{\mathrm{K}_{1} \mathrm{e}_{1}}{\alpha_{1}}
$$

Where $\Theta_{1}=\beta_{1} \mathrm{f}_{11}\left(\gamma_{1} \mathrm{z}_{1}-\delta_{1} \mathrm{y}_{1}\right)+\beta_{1} \mathrm{x}_{1}\left(\gamma_{1} \mathrm{f}_{31}-\delta_{1} \mathrm{f}_{41}\right)$

$v_{2}=\frac{\Theta_{2}}{\alpha_{2}}-f_{42}-\frac{\mathrm{K}_{2} \mathrm{e}_{2}}{\alpha_{2}}$

Where $\Theta_{2}=\beta_{2} \mathrm{f}_{12}\left(\gamma_{2} \mathrm{z}_{2}-\delta_{2} \mathrm{y}_{2}\right)+\beta_{2} \mathrm{x}_{2}\left(\gamma_{2} \mathrm{f}_{32}-\delta_{2} \mathrm{f}_{42}\right)$

$v_{3}=\frac{\Theta_{3}}{\alpha_{3}}-f_{43}-\frac{\mathrm{K}_{3} \mathrm{e}_{3}}{\alpha_{3}}$

Where $\Theta_{3}=\beta_{3} \mathrm{f}_{13}\left(\gamma_{3} \mathrm{z}_{3}-\delta_{3} \mathrm{y}{ }_{3}\right)+\beta_{3} \mathrm{x}_{3}\left(\gamma_{3} \mathrm{f}_{33}-\delta_{3} \mathrm{f}_{43}\right)$ 
$v_{4}=\frac{\Theta_{4}}{\alpha_{4}}-f_{44}-\frac{\mathrm{K}_{4} \mathrm{e}_{4}}{\alpha_{4}}$

Where $\Theta_{4}=\beta_{4} \mathrm{f}_{14}\left(\gamma_{4} \mathrm{z}_{4}-\delta_{4} \mathrm{y}_{4}\right)+\beta_{4} \mathrm{x}_{4}\left(\gamma_{4} \mathrm{f}_{34}-\delta_{4} \mathrm{f}_{44}\right)$

The error dynamical system simplifies to:

$$
\begin{aligned}
& \frac{d^{q} e_{1}}{d t^{q}}=-K_{1} e_{1} \\
& \frac{d^{q} e_{2}}{d t^{q}}=-K_{2} e_{2} \\
& \frac{d^{q} e_{3}}{d t^{q}}=-K_{3} e_{3} \\
& \frac{d^{q} e_{4}}{d t^{q}}=-K_{4} e_{4}
\end{aligned}
$$

Next, we consider the lyapunov function as:

$$
\mathrm{V}(\mathrm{e}(\mathrm{t}))=\frac{1}{2}\left(e_{1}{ }^{2}+e_{2}{ }^{2}+e_{3}{ }^{2}+{e_{4}}^{2}\right)
$$

Differentiating we get

$$
\begin{gathered}
D^{q}(\mathrm{~V}(\mathrm{e}(\mathrm{t}))) \leq \\
\left.=-K_{1} e_{1}{ }^{2} D^{q} e_{1}+K_{2} e_{2}{ }^{2}-e_{2} D^{q} e_{2} e_{3}{ }^{2}-e_{3} D^{q} e_{4}{ }^{2}+e_{4} D^{q} e_{4}\right) \\
<0
\end{gathered}
$$

i.e. $\mathrm{V}(\mathrm{e}(\mathrm{t}))$ is a positive definite function with a negative definite derivative. Hence, by Lyapunov Stability Theory we have that error tends to zero, implying desired antisynchronization has been achieved.

\section{Conclusion}

In this paper four hyper-chaotic fractional order systems have been synchronized in compound difference anti-synchronization manner by designing suitable controllers. This technique will find application in secure communication, control systems etc.

\section{Acknowledgment}

The second author is supported by C.S.I.R., India sanction letter no. 09/466(0189)/2017EMR-I by their Junior Research Fellowship.

\section{References}

1. L. M. Pecora and T. L. Carroll, Phys. Rev. Lett. 64, 821 (1990). https://link.aps.org/doi/10.1103/PhysRevLett.64.821

2. A. Khan and P. Trikha, Ind. J. Indust. Appl. Math. 8, 118 (2017). https://doi.org/10.5958/1945-919X.2017.00010.X

3. A. Khan and P. Trikha, SN Appl. Sci. 1, 757 (2019). https://doi.org/10.1007/s42452-019-0776$\underline{\mathrm{x}}$

4. J. Sun, Y. Shen, and G. Cui, Adv. Math. Phys. 2015, ID 921515 (2015). https://doi.org/10.1155/2015/921515

5. A. Khan, D. Khattar, and N. Prajapati, Pramana 89, 90 (2017). https://doi.org/10.1007/s12043$\underline{017-1488-7}$ 